\title{
How a Painter Paints: An Interdisciplinary Understanding of Embodied Creativity
}

\author{
Suk Kyoung Choi \\ Simon Fraser University \\ 8888 University Dr Burnaby BC \\ Canada V5A 1 S6 \\ choisukc@sfu.ca
}

\author{
Steve DiPaola \\ Simon Fraser University \\ 8888 University Dr Burnaby BC \\ Canada V5A 1 S6 \\ sdipaola@sfu.ca
}

\begin{abstract}
Tacit knowledge represents an embodied understanding of experience that shapes our form and action (Polanyi). For this study, the mimetic transformation of this 'hidden' knowledge is described as a process of active engagement in order to arrive at a conditional representation of experience that seeks validation through the stimulation of intersubjective 'resonance'.
\end{abstract}

Embodied cognition. Art painting. Metaphor. Intersubjectivity. Enactive transformation. Perception. Semiotics.

\section{INTRODUCTION}

How may we access, represent and preserve the lived experience tacitly embodied in the artist? How may one begin to describe the continuous process of creativity? Artists investigate these questions every day. Their answers lay in their work and it is to these exuvia of expression, the artefacts of experience, which we turn to for our data.

Drawing from the seminal definition of the principles of abstract art posed by Wassily Kandinsky in the early years of the twentieth century, and contextualizing that knowledge in contemporary cognitive metaphor theory (Lakoff \& Johnson, 1999), the study attempts to arrive at an understanding of how cognitive structure emerges in pragmatic self-observation during the artistic process. Through application of the concept of "enaction" (Varela, Thompson, \& Rosch, 1991), the research proposes a first-person approach to the exploration and derivation of the syntax of interactive creativity.

We attempt to define and visualize (see figures) a cognitive framework capable of representing the artist's pre-expressive state (the tacit) and its relationship to the final realized artwork on canvas (the trace). An artist's embodied experience constitutes an experiential understanding of interactive creativity, an understanding that takes as its foundation the practice of intentional expression and reflective self-observation of contextual response (Fig. 1, Fig. 2).

This creative conceptual engagement of expressive and reflective perspective constitutes process in the act of painting (e.g. Kandinsky; Dewey; Arnheim). The polarities of this process define a space of interaction that begins with the latent intentionality of the pre-expressive moment of the artist and ends with the creative artefact (e.g. Bloom; Levinson; Kornblith).

The artist's embodied experience therefore presents a lived, if unspoken, understanding of how spontaneous variation emerges in the creative act through an embodied shaping of conscious thought by the cognitive unconscious (Baars, 1988; Lakoff \& Johnson, 1999). This framework may be used to represent the spatial and temporal dimensions of the artistic impulse in the procedural space of an emergent computational grammar of artistic process (Fig. 5).

In our prototype system which outputs visualizations of creative process, we investigate this act and artefact pairing between a fine art painter and the realized painting through a comparative analysis of the discourse around process and the emergent artefact of that process. It is hypothesized that the artist's engagement within this framework forms a discernibly continuous and dynamic modelling of the tacit. 


\section{ENACTIVE TRANSFORMATION}

"The validation of epoche is the intuitive awareness of a deeper question." (Choi, 2013)

This research takes the position that creative process constitutes a phenomenology of intuitive practice, and that its central concern is not the making of objects but the understanding of experience and the making of meaning. This view of process involves a cycle of epoche leading to intuitive evidence, a technique of self-reflexive investigation (Fig. 3) aimed at recognizing the appearance of the immanent creative and the looking into the source of its origins. The technique involves a first-person suspension of belief followed by redirection, acceptance, and a reductive examination of essential meanings of expression iterated over an extended period of time in order to arrive at an intersubjectively verifiable experience.

\section{An example, from SK Choi:}

"I am teaching a group of students how to draw an apple. That is what they think. I am more interested in teaching them how they can learn to see the apple. A side interest is in guiding them to the realization that materials can be used for anything. I cannot possibly take over control of every (or any) neuronic and cellular pathway engaged in the observation, processing, reflection, and somatic expression of the response to that observation. Every line drawn is a new line. The only contact point I have is through metaphor. So the question becomes what do you see? But more importantly, what is there to be seen?"

\begin{abstract}
"I imagine that some of them might see a common fruit. What is that? It seems so be 'redness' clustered with 'roundishness' and some 'undrawable' association with taste. If drawn from imagination, it always has a stalk. There are individual variations that have as much to do with the student's interest as with any perception, or, more correctly, perception arises from interest, accurate perception rises from reflective consideration. Some of them just see an assignment, or a supermarket."
\end{abstract}

Draw what? The students stare with blank faces; the enormity of the question of what to draw. A sense of narrative lays in wait in the line. We even call them 'lines' of poetry, lines drawn out from an unknown source, describable only through metaphor. Drawn out from where? The mystery of the creative process is perhaps most generally represented as in Figure 1.

The research examines the cognitive experience contained within the embodiment of the difference between the continuous and the repetitive. It examines the problem of translation from one media to another and what is the nature of 'a medium' that meaning must pass through, from one human to another.

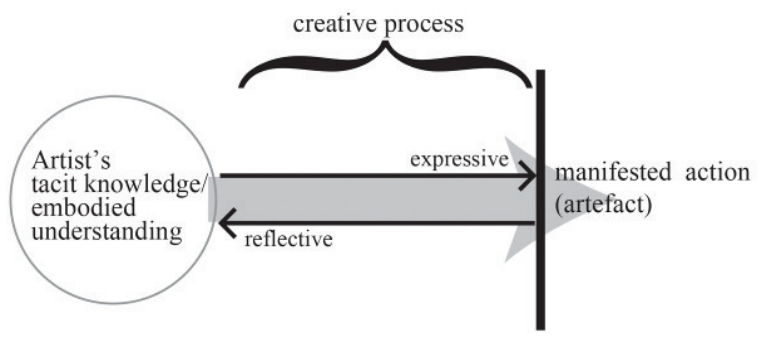

Figure 1: Creative process (Image @ Suk Kyoung Choi, 2012)

\section{1 'Having' feelings}

A key dimension of the research problem is the balance between qualitative and quantitative mediation, or in the terms of this research, the perceptual distinction between the continuous and the sampled: one infinitely rich but elusive, the other suffering the unbearable loss of experience with every translation. These questions may be posed as problems of neuroesthetics, which we will define in terms of cognitive science as the study of the code of art. In translation from perception to the trace of expression, if it is not possible to describe the latent potentiality of the former then it is not possible to meaningfully construct the distinctions made by the latter. An enriched depth of understanding enables more complete descriptions of experience. It is not the accuracy of the translation (an accurate description, if it could be obtained, can be accurately translated) but the metaphoric depth of the intersubjectively felt experience that is so variant and essential in this esthetic.

We will present a short demonstration of an approach to a problem by way of a thought experiment about technology. That experiment begins with a thought of Kandinsky's, that metaphors of form go far beyond the confines of the picture plane (Kandinsky, 1979, p. 13).

The problem? Perhaps the problem is more felt than stated, but an attempt may be made to describe it in terms of analogy, a procedure that shall prove central to the proposition of arriving at a model of intersubjective understanding. We start with a simple question:

\section{What is texture?}

Immediately we encounter a metaphor in the spontaneous response; texture is feeling. Now imagine a mixture of raw meat and leaves, some sand, paper, and a pomegranate all squished together under glass. What is the feeling? And if the glass becomes a branded interactive touch surface, what is the feeling? At what point do we 
lose sight of the flesh and believe the image? Where is the texture and how does it change? For the artist, these questions go far beyond information requisite to physical survival yet are perceived through common eyes and as far as we know, similar brains, formed in the gravitational well and biosphere of Earth, in one of a number of human cultures and contexts. What is seen, the categorized, the divided (in Deleuze's terms, the striated) is a small subset of what is seeable. Time and scale play a part in perception. According to Richard Gregory (1980), perception is $90 \%$ memory-based hypothesis, a suggestion which speaks directly to the depth of embodied cognition. Yet memory is surely tied up with emotion. Richard Cytowic identifies the limbic (sometimes called the "emotional brain") as the central determinant of the salience of perceptual information (Cytowic, p. 213).

I have chosen the term texture in order to model a cognitive bridging between these dimensions of embodied experience within the process of painting.

Richard Wollheim suggests that a proper account of pictorial meaning is found not in conventions and symbol systems, but in the non-propositional psychological account. This necessarily leads to an intersubjective stance whereby "what a painting means rests upon the experience induced in an adequately sensitive, adequately informed, spectator by looking at the surface of the painting as the intentions of the artist led him to mark it" (Wollheim, 1987, p. 22, emphasis added). By Wollheim's account, "ways of painting pair with kinds of intention" (Wollheim, 1987, p. 18). In other words, creative artefact production encodes intention. This study asks in what ways may that looking make available to the first-person the experience of another?

The artist draws out a mental image from within their experience and expresses it as something perceivable to others. What constitutes the mental image is a collection of experiences from deep memory to the immediate awareness of the world; from the unconsciousness to the immediate sensation of reality. Impressionist artist Claude Monet set himself the task of painting the subtlety of the constant change of light over the face of the Rouen Cathedral in order to demonstrate the impossible and indivisible juxtaposition of the ephemeral and the unmoved. How did Monet achieve this? Seemingly, through layer upon layer of iterative reflection upon the experience - the paintings are thick with the materiality of paint while radiating a sublime diffuse texture of light. The Rouen Cathedral series traces the movement of light, through the constant epoche of observation, 'stepping back' in time to illustrate that what is permanent about the place, after all reduction, is its constant change, and the journey of recording that realization in a way that may be felt by others.

There are many elements the artist observes, and while those observations are built upon previous experiences of the artist (the artist knows already what they are observing, such as how to understand colour, and how to perceive and categorize objects), the artist sees but does not look for what is already known. The artist looks for what has changed. What an artist observes must therefore be described through a language of interactive sensorial perception. The artefact forms the trace of the process. Cognitive neurobiologist Semir Zeki has proposed that the creative process of the artist constitutes a neurologically motivated pragmatic investigation of perception. But this investigation must identify, as Jonathan Gilmore, lecturer in philosophy at Yale University reminds us "what is distinctive in our experience of art, how art carries meaning_material, contextual, intentional, or historical-or how art contributes to experience, extending sensation, rather than just replicating it" (Gilmore, 2006).

Individual experience is not separable from empathic response. Therefore a neuroesthetic investigation of creative action is not concerned with demystification of the visceral so much as it is with its abstraction. This may be represented in a balanced way (Fig. 2).

The epoche of expression and reflection may be represented as a cycle where the enactive metaphor within empathic response is in opposition to the artefact. Enactive process has what may be thought of as an input and output phase. Observation constitutes a sensorial accumulation of embodied meaning, called here 'sensorial metaphors'. These sensorial metaphors attempt to place perceptual experience in intersubjectively communicable categories. Thus we have shared understandings like what the colour red looks like, or what the scent of an apple might be.

The input phase is associated with the contextually observed conditions, the subject, or perhaps as in Monet's Rouen series, 'the real light'. Various observations may be made about this light. It is the painter's work to translate the experienced world through an experiencing body.

To the external observer, the first appearance of the act of painting is directly associated with action; expression is most essentially a body in motion. In order for this expression to convey any intersubjective meaning, that expression must be framed in terms of shared somatic metaphors. These somatic metaphors form a template for a way of moving in a continuous appropriate 
response to the intuitive translation between the observer and the artist's expression.

We then have a model of process that associates a conceptual translation from the input data of sensorial metaphors with a balancing response of expressive output data of somatic metaphors (Fig. 2). Intuitive translation occurs in the interconnection between the sensorial metaphor and the somatic metaphor.

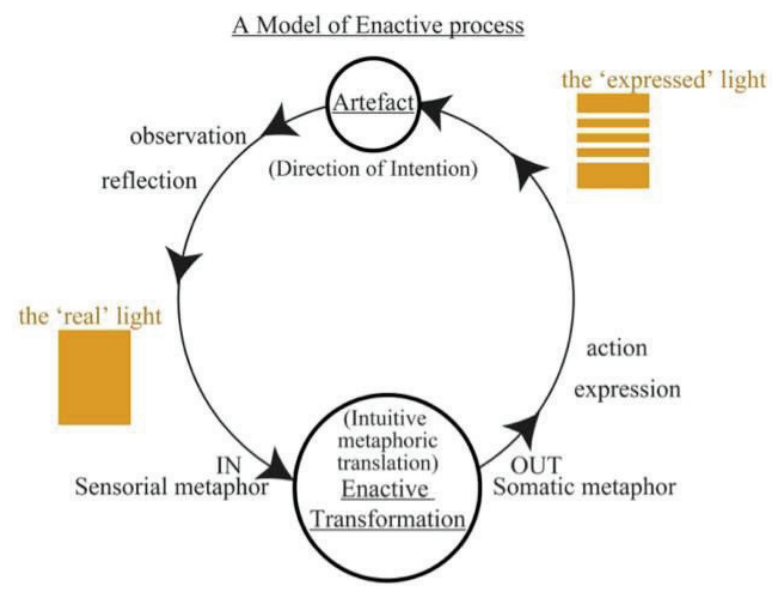

Figure 2: Metaphorical enactive transformation

Note. The translation of sensorial reflection to somatic expression. Inspired by various studies of reflective process (Dewey, 1934/1958; Depraz, et al., 2003)

(Image (C) Suk Kyoung Choi, 2012)

In a model of process that admits of continuous awareness, where the physical informs the immaterial through some mysterious interface labelled "the brain", it would be difficult to argue for the divisibility of the visceral from the "strictly cognitive'. We therefore utilize (after Lakoff \& Johnson) the trace of embodied metaphor in probing this interface in order to investigate the question of in what manner and to what extent, in an enactive model of cognition, the artefact that forms experience may reflectively encode the trace of the passing of the artist's mind.

\subsection{What does the artist see?}

Michael F. Land has noted that almost all studies of vision have dealt with eye movements as singular events. Saccades, stabilizing reflexes, pursuit and vergence have for the most part not been modelled as coexistent operations of a unified cognitive strategy (Land, 2006, p. 300). As he points out this tends towards the view that eye movements are very task specific and momentary (Land, 2006, p. 322 ), their functional relevance to immediate needs takes precedence over image statistic sorting. In other words, the eyes are directed towards some imagined (intentional) object. Eye movement seems fundamental to a large portion of the work of the artist. It is both intentional and reactive.
But when the artist paints, they do not see only the point. Kandinsky proposed what was essentially an enactively derived model of cognition and revolutionized the image in Western culture. The schematic source of all that is observable is contained in Kandinsky's Point. The point follows a journey from "the briefest, constant, innermost assertion: short, fixed and quickly created" to it's "breaking free of the tension containing it" whereupon the line, the path of the moving point, is born. Kandinsky's view of perception is idealisticholistic. A reflective creative act does not occur without embodied reference to the spatial and emotionally resonant interaction with the context of the event. At about the same time in the history of western models of perception, Picasso's development of analytic and synthetic cubism brought multi-dimensional space-time and sampling into the domain of visual representation.

Zeki identifies a fundamental cognitive property of constancy, whereby "the function of the visual brain is to seek knowledge of the constant and essential properties of objects and surfaces, when the information reaching it changes from moment to moment" may be imagined as patterns in time. The artefacts are dimensional sections of experience, landmarks on a journey.

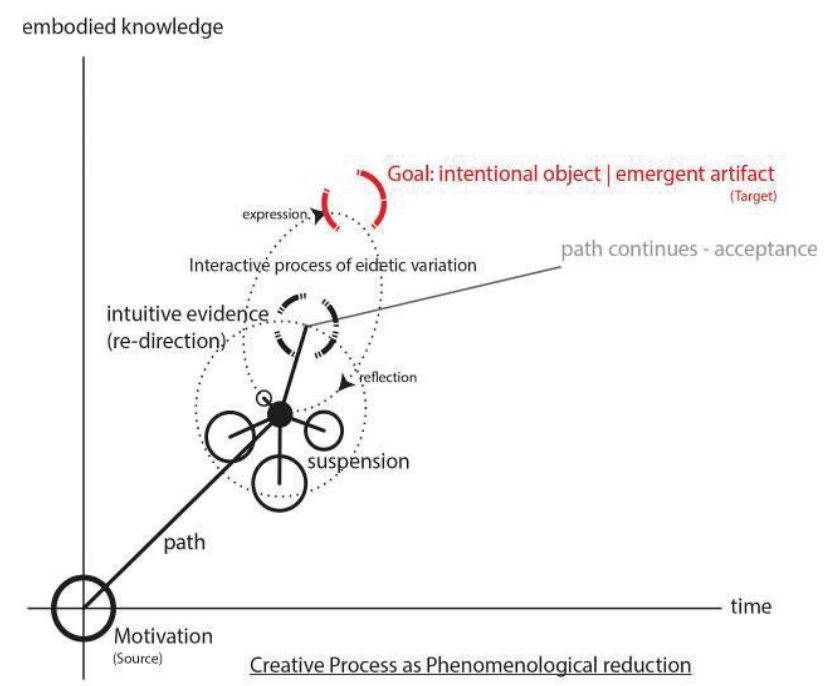

Figure 3: Source - Path - Goal

Note. Creative Process as Phenomenological Reduction: Intuitive evidence initiating redirection. Inspired by metaphorical image schema (Johnson, 1987; Lakoff, 1987/1990). (Image @ Suk Kyoung Choi, 2012)

The trace of the creative pursuit of the intentional object is represented schematically in Figure 3. This is a process of epoche over constant iteration; motivation, suspension of belief, searching for evidence that the goal is near, redirecting and letting go of the new belief. The artist's goal is not what is recorded but the direction latent within intention. Mark Johnson and George Lakoff 
suggest that the Source-Path-Goal schema is one of the most common structures that emerge from constant body function. This embodied schematic directionality is essential to the notion of texture. The point, a directionless 'tension', begets the directionality of the line, which in turn begets the shape, forming patterns in space and in time.

\subsection{The Metaphor of Texture}

Artist-cognitive scientist Steve DiPaola notes, “... portrait artists over 1000's of years have somewhat intuitively evolved a 'painting methodology' which exploits specific human vision and cognitive (neural) functions" (DiPaola, 2008).

Amy lone, writing about the role of the artist in studies of perception and consciousness, stresses that the "artist's activity is both active and cognitive" (lone, 2000). Artists have always represented this process through pragmatic experimentation; by reducing the size of distant objects, dulling down colours, or blurring details as the object gets farther away. But all these techniques are descriptions, accounts, of an event that is experienced through translation; they are metaphors of experience.

We investigate the motivations of creativity that result in intersubjectively observable phenomenon and that leave behind the artefact, asking whether intention, drawn from the embodied understanding that is the artist's world, might not be expressed somatically through the entire neural network of the human being. If expression occurs at minute levels of granulation, then its resonance must be observable in diverse transcriptions of the event at varying scales.

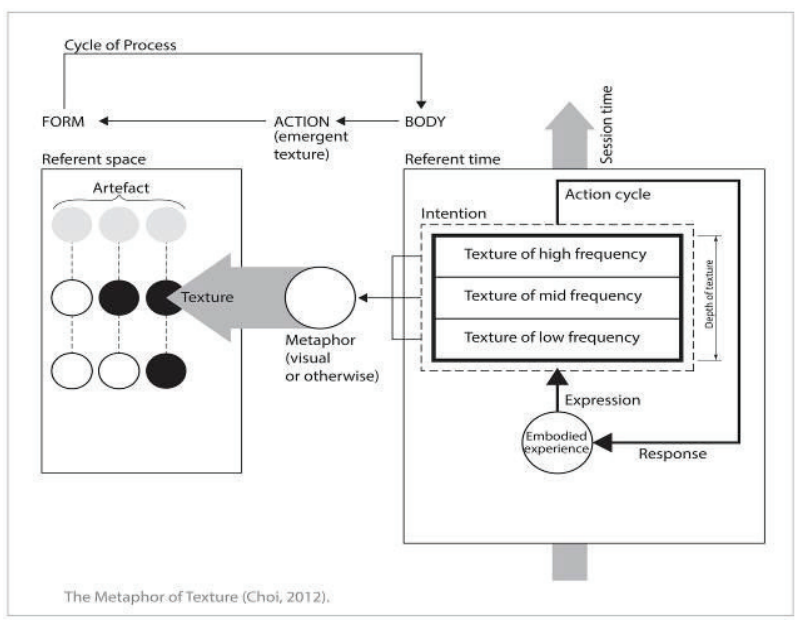

Figure 4: The Metaphor of Texture - Multi-temporality

Note. Frequency and session time intersect at the interface metaphor. (Image (c) Suk Kyoung Choi, 2012)

Figure 4 attempts to model a multi-temporal description of texture that is inclusive of an iterative and enactive relationship with the artefact through the intentional object; the metaphor of the interface. It is the intent of intention, if you will, to exist transparently with the 'other', the object of consciousness. But the enactive ontology places a third entity in this relationship, that of dynamics: action is required for interaction, a change of state implies motivation. For the body to interact with objective form, to create form, it is embodied that there must be movement. There is then, within the essence of the first-person, already an emergent certainty of the objective. One cannot have certain kinds of experiences without intentional somatic actions. This experience, in Johnson and Lakoff's terms, is perceived as 'outside the self' in their cognitive model of the Container schema, a schema so fundamental to human life. This dynamic transformation of subject and object occurs neither in the purely somatic space of the body's expression, nor purely the sensorial space of reflection, but in a metaphoric space of what I have called, after Varela, Thompson, \& Rosch (1991), enactive transformation. It is in this space where texture is formed (emerges). Texture is a residue of interaction.

So in this model, the body contains a record of time and the artefact, a record of space. The body is a skin bound container of autonomous changes. Over time, various intentional experiences accumulate and are embodied through an action cycle of expression and response.

The framework proposes a model of metaphoric texture accounting for relationships between embodied action, the relationship to the object of intention, and the multiple temporalities embedded in the process of formation of the artefact.

In painting, "texture" is constituted of all the dimensional elements, point, line, and plane, composed through the various metonyms of form by an intentional salience that is the first-person. We add to these structural elements a consideration of Lakoff and Johnson's metaphoric schema as applied to an examination of creative process in painting, to account for texture's embodied intentionality. Lakoff and Johnson argue that cognition is based on manipulation of schemas, or sets of metaphorical understandings about the world that are grounded in fundamental physical spatial relationships (Johnson, 1987). These schemas therefore constitute the embodied grounding of what the artist can do, but which they find difficult to describe. These schemas map the texture of creative experience.

By this theory, there are intentions expressed through art that are imbued in the artefact as the 'essence' of the work. Vilayanur Ramachandran presents a "coherent biological framework" in his Eight Laws of Artistic Experience that proposes to 
explain esthetic response (Ramachandran, 1999, p. 17). It is pertinent to note the essentially enactive foundation of the perceptual rules employed in the framework. Esthetic response grows from the same embodied understanding of context as does the need to survive, leading Ramachandran to ask what may be the "survival value of art" (p. 16).

Cristoph Redies proposes a universal biological model of visual esthetic perception (Redies, 2007). The model accommodates "the central tenet of neuroesthetic theory that esthetic perception reflects fundamental functional properties of the nervous system", suggesting a level of embodied visual schema are at play at abstract levels of cognition. Redies argues that it is the goal of artistic practice to create a "composition that is able to put his own nervous system into a particular functional state (resonance)". In other words, composition can induce the specific neural states in the artist's nervous system. This suggests that it may be possible that an intersubjective resonance arises in the viewer of that work, accounting for the degree to which meaning is shared about a particular artefact by multiple viewers, and potentially revealing cross-cultural metaphorical constructs.

From this theoretical basis, then, there begins to emerge the foundations of a method of categorization of domains of significance to painters. If "perception and action, sensorium and motorium, are linked together as successively emergent and mutually selecting patterns" then significant domains should reflect the intersubjective processual relationship between form and action (Varela et al., 1991, p. 163). In the texture of the painting reside both the painting and the painting of the painting.

Texture, then, is an embodied seeing from multiple points of view, and those points of view include the empathic response that is entirely emergent.

\section{APPROPRIATE METHODS}

Every question has multiple answers. The question should not presuppose the answer but initiate a series of research events that move through interconnected domains of distinction that emerge through constant reduction of the question. This iterative approach will be essential to progression in the first-person reduction of experience.

In the sense offered by Gregory, that 'perceptions are hypotheses', the conceptual diagram forms a self-reflective memory map of process whereby process turns in on itself to 'observe observation'. This is a primary function of the practice of the artist and establishes strong grounds for validation of art-as-research in disciplines concerned with the study of self-awareness.

Preliminary work with this model has suggested that cross-domain metaphor is frequently encountered in sessions of reflexive pragmatic activity such as painting (Choi, 2013). This is taken as an intimation of active semiotic transformation at pre-conscious levels of cognition (Cytowic, 2002). Our conceptual model therefore suggests that knowledge is derived and encoded by pattern matching across sensorial domains. In perception, an apple is never just a 'red roundishness', but a complex texture of experiences embodied as "an apple", including changing colours, weights and subtle scent memories. It is frankly astonishing that out of the essentially endless barrage of sensorial data we are able to find one thing to eat, or draw. This property of consciousness to find and ascribe meaning in ways that reflect the rapid application of embodied abstract representations of known states, from the endless variation presented to us, has been identified by Zeki as 'essential and primordial functions of the visual brain' (Zeki, 2001).

Conceptual models are limited by their assumed parameters. One can observe metaphors in expression, but it is a different thing to observe their reflection. So in this model (Fig. 5), which was derived in a series of recorded and self-reflective videos of a series of painting sessions, this problem occurs at the point of 'metaphoric translation'. The richness of this conduit is somehow limitless yet defining: The problem with categories is that even to sort by something so apparently fundamental as Zeki's "constancy" and "abstraction" immediately requires experiential and cognitive definitions of what exactly is "constancy". So in these conceptual models of embodied process it is considered a more successful abstraction if the categories themselves are adaptable to diverse schema yet whose members situate as close to intersubjectively identifiable 'qualia' as conceivable. The research problem is in any case immense. So we began to wonder, whether resonant patterns in the artefact, as identified as possible by Redies, might not be intersubjectively observable by means that could likewise account for the play between the continuous and the repetitive nature of the medium that was represented by the artefact but which we are modelling as a temporal accumulation of residual activity we call texture.

A visualization of process presumes it is possible to find intersubjectively verifiable links between the discourse around the process, the associated actions, and the artefact generated by that process. Taking a lead from Marshall McLuhan, we turn to alternate readings of the medium in order to more 
fully understand the nature of the message of texture.

As direct translations are artificial ones (in a continuous space) we propose an investigation through analytic comparison of perceptual space re-mapping. Utilizing the domains of experience proposed in the model (Figure 5), algorithms are developed that allow for non-visual readings of visual experiences. For example, the trace of the viewer's gaze over a work of art, presumably following to some extent the encoded intention of the artist, is translated into audio representations of the field of view utilizing adaptive localized sorting algorithms derived from first-person practice. These first-person empathic experiences might be examined for data resonances that occur across different viewers of the same work. An enactively derived investigatory position in a cognitive science of creativity, as Jonathan Gilmore notes, must acknowledge how art extends sensation. Another avenue of interest is to what degree sensation is encapsulated in the artefact of process: is it possible that micro-structures in material resonate with and 'remember' intentions that the artist themselves were barely aware of? A criticism may be that this is a bit like looking for needles in a haystack, but with the advent of computational biometric interaction with the creative process in a neuroesthetic practice of art, those needles are becoming increasingly possible to find, if only we remember to reflect on the mystery of losing them in the first place.

\section{CONCLUSIONS}

We have presented a speculative framework of interrelated views of perception that feed into the development of an interdisciplinary understanding of interactive creativity. This preliminary investigation seeks to encompass the perspectives of a wide range of disciplines engaged in cognitive research in order to inform the modelling of an interactive shaping of experience. We have set a bracketing around the tacit that ranges from the continuous state of abstraction to the granulation of individuated qualia.
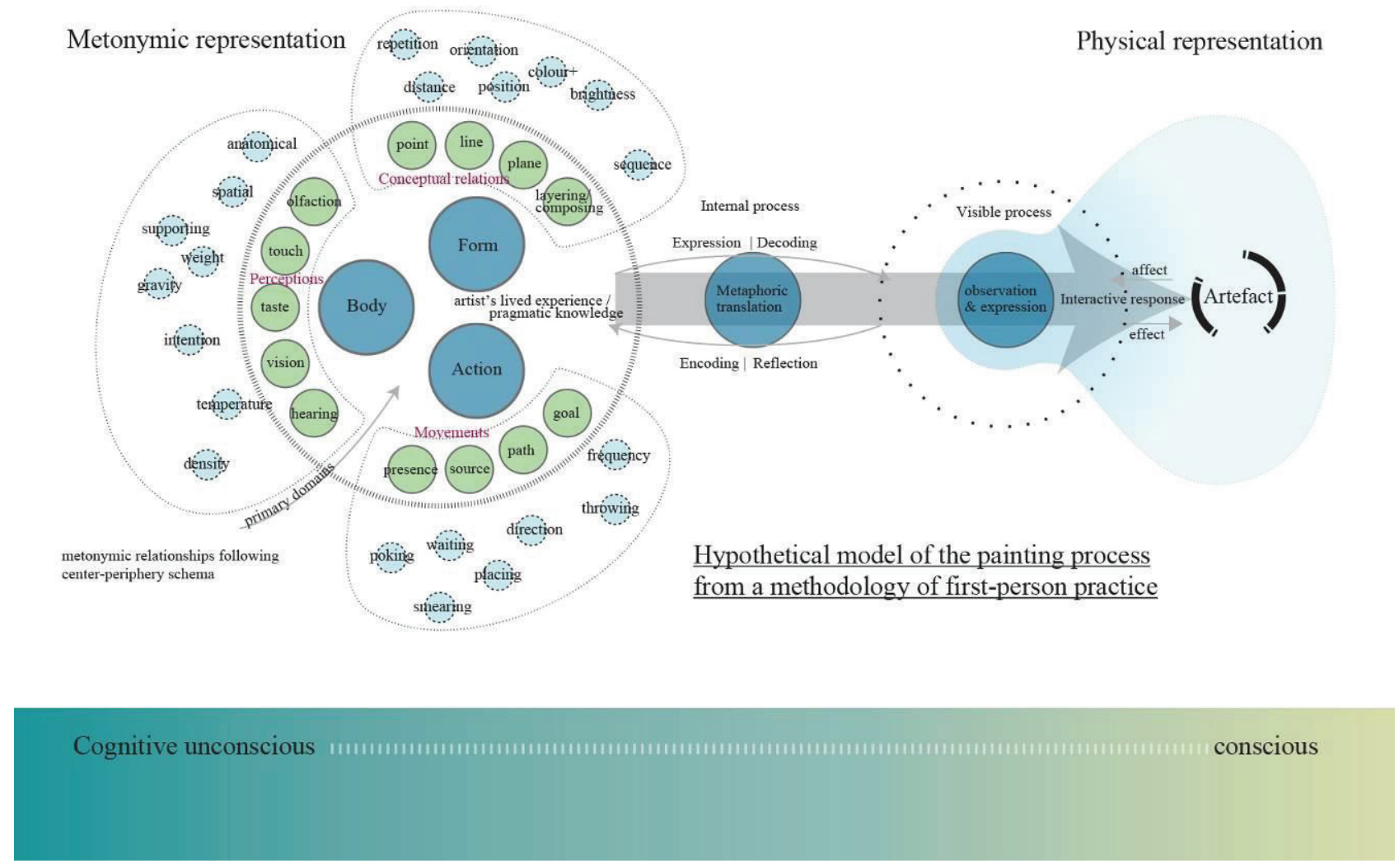

Figure 5: Experiential domains of metaphor

Note. Process as a continuum of enactive structures between unconscious embodied knowledge and intentional objects. The model may be extended by application to (and by drawing from) other disciplines and practices. (Image @ Suk Kyoung Choi, 2012) 
A growing need to acknowledge the cultural crisis of "losing touch" in a mediated world where visual primacy is assigned to the touchless 'touch' screen motivates this study. Knowledge is being lost at an alarming rate as embodied experience falters at the digital threshold of a brave new world. As painful experience is replaced by the soma of the App, and as perception is replaced by vision, will we still remember how to touch?

\section{REFERENCES}

Arnheim, R. (1954/1974) Art and Visual Perception: A Psychology of the Creative Eye. University of California Press, Berkeley, CA.

Baars, B. (1988) A Cognitive Theory of Consciousness. Cambridge University Press, Cambridge and New York.

Choi, SK. (2013) Painting Time: The metaphor of texture. MA thesis, Simon Fraser University, BC. Available at https://theses.lib.sfu.ca/thesis/etd7650

Cytowic, R. E. (2002) Synesthesia: A Union of the Senses (2nd Edition). MIT Press, Cambridge, MA, USA.

Deleuze, G., and Parnet, C. (1977/1987) Dialogues. (Hugh Tomlinson and Barbara Habberjam, Trans.). Columbia University Press, New York. (p vii).

Dewey, J. (1934/1989) "Art as Experience". Reprinted in John Dewey: The Later Works, 19251953, Vol. 10. Boydston, J. (ed.). Southern Illinois University Press, Carbondale.

Dewey, J. (1929/1958) Experience and Nature. Open Court Pub. Co., La Salle, III.

DiPaola, S, (2009) Exploring a Parameterized Portrait Painting Space, International Journal of Art and Technology, Vol 2, No 1-2, pp 82-93.

Gilmore, J. (2006) Brain Trust: On art and the new biology of mind. Artforum International, Vol. 44, No. 10 pp. $121-122$.

Gregory, R. L. (1987) Perceptions as Hypotheses. Philosophical Transactions of the Royal Society of London B, 290, pp. 181-197.

Gregory, R. L. (1966/1997) Eye and Brain: The psychology of seeing. Princeton University Press, Princeton, N.J.

Ione, A. (2000) An Inquiry into Paul Cezanne: The Role of the Artist in Studies of Perception and Consciousness." Journal of Consciousness Studies, 7, No. 8-9, pp. 57-74.
Johnson, M. (1987) The Body in the Mind: The Bodily Basis of Meaning, Imagination, Reason. The University of Chicago Press, Chicago.

Kandinsky, W. (1947/1979) Point and Line to Plane. Dover, New York, NY.

Lakoff, G., and Johnson, M. (1999) Philosophy in the Flesh: The Embodied Mind and Its Challenge to Western Thought. Basic Books, New York, NY.

Lakoff, G. (1987/1990) Women, Fire, and Dangerous Things: What categories reveal about the mind. University of Chicago Press, Chicago and London.

Land, MF. (2006) Eye movements and the control of actions in everyday life. Progress in Retinal and Eye Research 25 pp. 296-324.

Levinson, J. (2007) "Artworks as Artifacts". In E. Margolis \& S. Laurence. (Eds.). (2007). Creations of the Mind: Theories of artifacts and their representation. (pp. 74-82). Oxford University Press, Oxford and New York.

Margolis, E., and Laurence, S. (Eds.). (2007) Creations of the Mind: Theories of Artifacts and Their Representation. Oxford University Press, New York.

Miall, R. C., and Tchalenko, J. (2001) A Painter's Eye Movements: A Study of Eye and Hand Movement during Portrait Drawing. Leonardo, 34-1, (pp. 35-40): The MIT Press.

Polanyi, M. (1983) The Tacit Dimension. Peter Smith, Gloucester, MA.

Redies, C. (2007) "A Universal Model of Esthetic Perception Based on the Sensory Coding of Natural Stimuli." Spatial Vision 21 (1-2): 97-117.

Varela, F., Thompson, E., and Rosch, E. (1991/1999) The Embodied Mind: Cognitive Science and Human Experience. MIT Press, Cambridge.

Ramachandran, V., and Hirstein, W. (1999) The Science of Art A Neurological Theory of Aesthetic Experience." Journal of Consciousness Studies, 6, No. 6-7, pp. 15-51.

Wollheim, R. (1987) Painting as an Art. Thames and Hudson, London.

Zeki, S. (2001) Artistic Creativity and the Brain. Science 6. July 2001: Vol. 293 no. 5527 (pp. 5152): DOI: $10.1126 /$ science. 1062331 